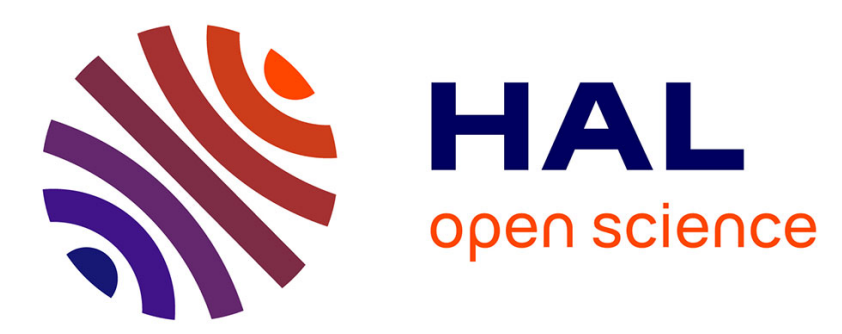

\title{
Raman-Brillouin scattering from a thin Ge layer: Acoustic phonons for probing Ge/GeO2 interfaces
}

Lama Yaacoub, Sylvie Schamm-Chardon, N.N. Ovsyuk, Antoine Zwick, Jesse

Groenen

\section{- To cite this version:}

Lama Yaacoub, Sylvie Schamm-Chardon, N.N. Ovsyuk, Antoine Zwick, Jesse Groenen. RamanBrillouin scattering from a thin Ge layer: Acoustic phonons for probing Ge/GeO2 interfaces. Applied Physics Letters, 2014, 104 (6), pp.061601. 10.1063/1.4864790 . hal-01720453

\section{HAL Id: hal-01720453 https://hal.science/hal-01720453}

Submitted on 1 Mar 2018

HAL is a multi-disciplinary open access archive for the deposit and dissemination of scientific research documents, whether they are published or not. The documents may come from teaching and research institutions in France or abroad, or from public or private research centers.
L'archive ouverte pluridisciplinaire HAL, est destinée au dépôt et à la diffusion de documents scientifiques de niveau recherche, publiés ou non, émanant des établissements d'enseignement et de recherche français ou étrangers, des laboratoires publics ou privés. 
Raman-Brillouin scattering from a thin Ge layer: Acoustic phonons for probing Ge/ $\mathrm{GeO}_{2}$ interfaces

L. Yaacoub, S. Schamm-Chardon, N. N. Ovsyuk, A. Zwick, and J. Groenen

Citation: Appl. Phys. Lett. 104, 061601 (2014); doi: 10.1063/1.4864790

View online: https://doi.org/10.1063/1.4864790

View Table of Contents: http://aip.scitation.org/toc/apl/104/6

Published by the American Institute of Physics

Scilight

Sharp, quick summaries illuminating the latest physics research 


\title{
Raman-Brillouin scattering from a thin Ge layer: Acoustic phonons for probing $\mathrm{Ge} / \mathrm{GeO}_{2}$ interfaces
}

\author{
L. Yaacoub, ${ }^{1}$ S. Schamm-Chardon, ${ }^{1}$ N. N. Ovsyuk, ${ }^{2}$ A. Zwick, ${ }^{1}$ and J. Groenen ${ }^{1, a)}$ \\ ${ }^{1}$ CEMES-CNRS and Université de Toulouse, 29 rue J. Marvig, 31055 Toulouse, France \\ ${ }^{2}$ V. S. Sobolev Institute of Geology and Mineralogy, Novosibirsk 630090 Russia
}

(Received 19 December 2013; accepted 28 January 2014; published online 10 February 2014)

\begin{abstract}
We report on Raman-Brillouin scattering by acoustic phonons from a thin Ge layer. The high frequency acoustic phonons involved in this scattering are used to probe the native oxide present on top of the Ge layer. By comparing experiment and photoelastic modelling, a quantitative analysis is performed which shows that an interfacial layer is located in between the Ge and $\mathrm{GeO}_{2}$ oxide layers. The native oxide is found to be composed of a $0.5 \mathrm{~nm}$ thick interfacial layer and a $1 \mathrm{~nm}$ thick $\mathrm{GeO}_{2}$ layer on top of it. Sensitivity down to the sub-nm scale is evidenced. (C) 2014 AIP Publishing LLC. [http://dx.doi.org/10.1063/1.4864790]
\end{abstract}

Germanium is gaining interest as an alternative to silicon for applications in electronics, owing to its higher carrier mobilities, lower operating voltages, and lower processing temperatures. However, to fully benefit from these interesting properties in devices, controlling the quality of the interface between $\mathrm{Ge}$ and its $\mathrm{GeO}_{2}$ oxide is a prerequisite. ${ }^{1}$ Both theoretical and experimental studies have been reported, aiming to achieve a detailed understanding of the electronic and structural properties of $\mathrm{Ge} / \mathrm{GeO}_{2}$ interfaces. ${ }^{2-5}$ Using Fourier transform infrared spectroscopy, it was shown that substoichiometric $\mathrm{GeO}_{x}$ transition layers are formed during oxidation in air ambient, prior to the $\mathrm{GeO}_{2}$ formation. ${ }^{2}$ The local structure around $\mathrm{Ge}$ atoms and electrostatic discontinuity at $\mathrm{Ge} / \mathrm{GeO}_{2}$ are usually investigated using X-ray photoelectron spectroscopy (XPS). ${ }^{3}$ Further insight into the atomic structure of these interfaces was gained by comparing valence band offsets derived from measured XPS shifts and calculated ones using hybrid density functionals. ${ }^{4,5}$ However, the actual bond pattern at the $\mathrm{Ge} / \mathrm{GeO}_{2}$ interfaces remains an open question. 5

In this Letter, we aim to demonstrate that Raman-Brillouin (RB) scattering by acoustic phonons is relevant for probing ultrathin germanium oxide layers. Since a few nm-thick oxide layer has no significant intrinsic RB signal, we investigate the Raman Brillouin scattering generated in a thin Ge layer on top of which the oxide layer is located. Acoustic phonons extending over the whole stack are sensitive to the Ge layer environment and thus, provide us with the required probing in the oxide layer. To probe at nm scale, high frequency acoustic phonons are required. Previous studies on RB scattering from quantum dots or thin films, ${ }^{6-8}$ have demonstrated that the more the electronic states involved in the RB scattering are localized, the more high frequency acoustic phonons do contribute to the spectra. Consequently, we have chosen a thin Ge layer $(25 \mathrm{~nm})$ to generate the RB scattering. Experimental data are analyzed by comparing with numerical simulations.

We investigate here the native oxide grown on the Ge surface. The Ge layer is deposited on $\operatorname{In}_{0.12} \mathrm{Ga}_{0.88}$ As which is

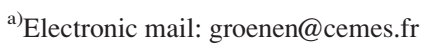

part of an InGaAs/GaAs (001) pseudosubstrate, initially designed for stress engineering. Introducing tensile biaxial strain in the Ge layer is an interesting path for enhancing carrier mobilities and band gap tuning. The $\operatorname{In}_{0.12} \mathrm{Ga}_{0.88} \mathrm{As}$ layer was grown up to $1 \mu \mathrm{m}$ thickness in order to ensure full elastic relaxation on its top and pseudomorphic subsequent growth of strained Ge. Details about the growth can be found in Ref. 9.

The Ge layer thickness $e$ was derived from atomic structure imaging with High Resolution Transmission Electron Microscopy (HRTEM). Imaging was performed with a field emission transmission electron microscope (FEI Tecnai F20) operating at $200 \mathrm{kV}$, equipped with a corrector for spherical aberration dedicated for the direct observation of atomic structures at interfaces with substantially reduced contrast delocalization in the images. From the image analysis, we deduced $e=25.5 \pm 0.5 \mathrm{~nm}$ (Fig. 1). The Ge oxide layer is also evidenced. Indeed, on top of the Ge layer, it appears as an amorphous layer which is darker than the glue. However, its top boundary not being well defined, it is difficult to determine its thickness with a sub-nanometer precision.

The Raman-Brillouin scattering was excited at room temperature using the $\lambda_{i}=568.2 \mathrm{~nm}$ line of a $\mathrm{Kr}$ ion laser. The scattered light was dispersed using a Horiba Jobin Yvon T64000 micro-Raman spectrometer, equipped with a CCD camera. Unlike for conventional micro-Raman measurements, the incident exciting laser beam and collected scattered light do not have any common path; the laser is focussed with an objective placed off-axis with respect to the collection objective. It avoids the reflected beam being directed into the spectrometer and Rayleigh scattering within the collection optics. According to the high refractive index of $\mathrm{Ge}(\mathrm{n}=5.34)$, we deal with an internal quasi-backscattering configuration.

The calculated RB spectra reported here were simulated according to the general formulation of photoelastic model presented in Ref. 7. This model includes the spatial modulation of the elastic, photoelastic, and optical properties. The scattering by longitudinal acoustic modes being polarized along the $z$ growth axis is calculated. All calculated spectra were Gaussian convoluted $\left(0.8 \mathrm{~cm}^{-1}\right.$ full width at half maximum), in order to account for the experimental resolution. 


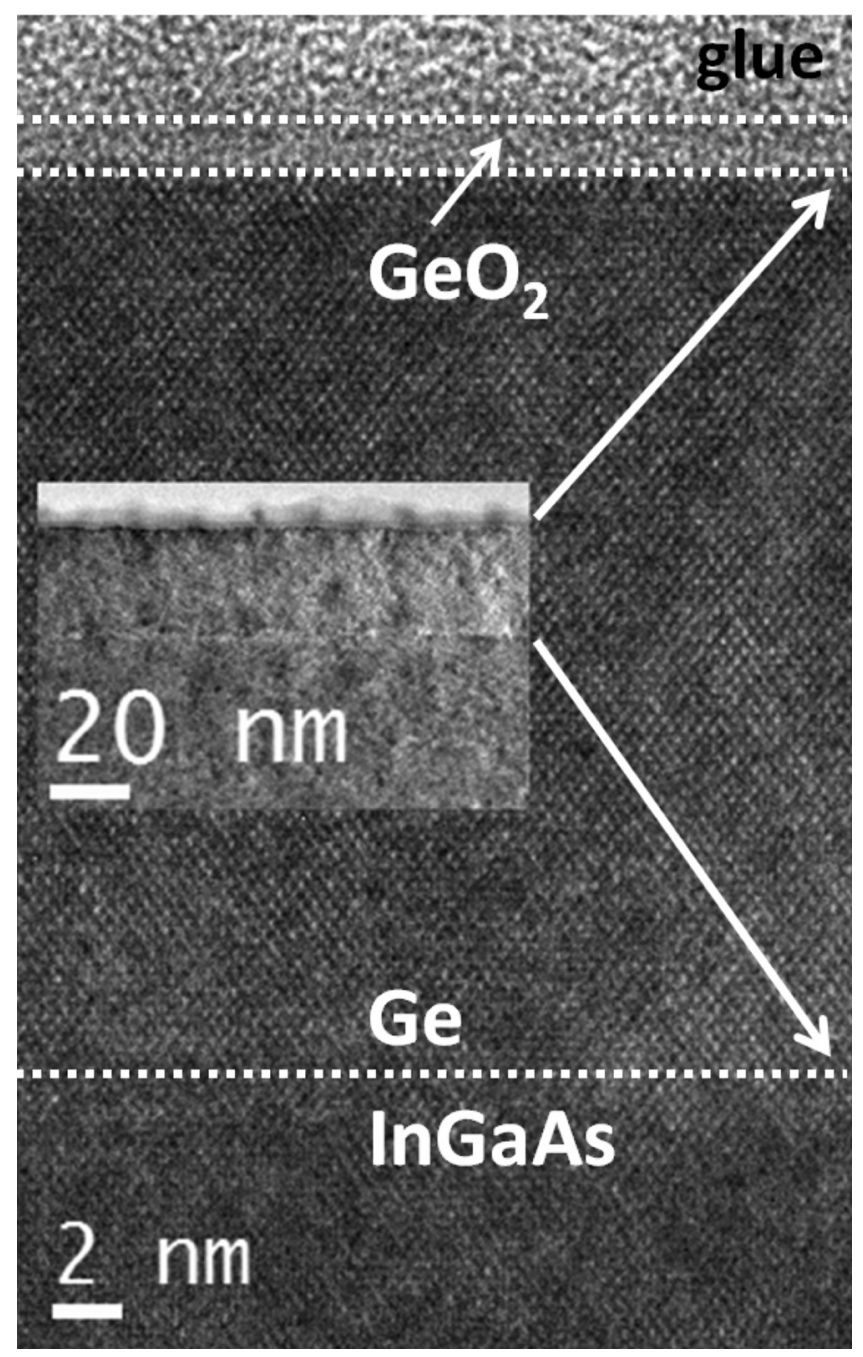

FIG. 1. HRTEM of the $\mathrm{GeO}_{2} / \mathrm{Ge} / \mathrm{InGaAs}\langle 110\rangle$ stack. TEM sample preparation glue is seen on the top of the stack.

Numerical values for sound velocities, mass densities, and optical indexes used in the simulations were taken from Ref. 10 for $\mathrm{Ge}$, InGaAs, and GaAs and from Ref. 11 for $\mathrm{GeO}_{2}$.

From our previous studies on single Si layers, we know that the $\mathrm{RB}$ response results from a complex interplay between acoustical cavity, optical cavity, and photoelastic effects. ${ }^{7,8}$ Interestingly, Ge and $\mathrm{In}_{0.12} \mathrm{Ga}_{0.88} \mathrm{As}$ are almost acoustically matched; their acoustic impedances (mass density times sound velocity) differ only by $1 \%$. Consequently, the $\mathrm{Ge} / \mathrm{In}_{0.12} \mathrm{Ga}_{0.88} \mathrm{As}$ interface does not form an acoustic cavity, facilitating significantly the analysis of the experimental data. Optical indexes being rather similar, optical cavity effects in the RB scattering are weak. At $\lambda_{i}=568.2 \mathrm{~nm}$, the Ge layer is selectively excited in resonance with the $E_{1}$-like transition of $\mathrm{Ge}$, providing a strong photoelastic response. On the contrary, the photoelastic effect within the ultrathin and transparent $\mathrm{GeO}_{2}$ oxide layer is negligible. Light absorption being strong in $\mathrm{Ge}$ at this wavelength and excitation being off-resonance for the InGaAs layer, one does not expect much signal originating from the InGaAs layer. Moreover, according to its $1 \mu \mathrm{m}$ thickness, the InGaAs RB signal contribution is a bulk-like Brillouin peak, being located below $5 \mathrm{~cm}^{-1}$. This spectral range will not be addressed experimentally here. We thus focuss on the RB originating from the Ge layer. Consequently, in the calculations, we assume the photoelastic effect to occur in the Ge layer only. We therefore define a photoelastic profile $p(z)$ with a constant value in the Ge layer and zero elsewhere. ${ }^{7}$

Let us first consider the simple case of the oxide free $\mathrm{Ge} / \mathrm{InGaAs} / \mathrm{GaAs}$ stack. The corresponding oxide free RB spectrum is reported in the bottom of Fig. 2. One observes a strong peak below $10 \mathrm{~cm}^{-1}$ and a series of regularly spaced peaks with rapidly decreasing intensity above. These peaks are not related to acoustic resonances in the Ge layer. Optical cavity effects being weak, one may consider a simplified version of the photoelastic model (See Eq. (2) in Ref. 7). Considering a simple standing acoustic wave resulting from the total reflection at the sample surface, the scattering efficiency as a function of wavevector $q$ is then proportional to

$$
\begin{aligned}
I(q) \propto & \left\{\operatorname{sinc}^{2}\left[(\Delta k-q) \frac{e}{2}\right]+\operatorname{sinc}^{2}\left[(\Delta k+q) \frac{e}{2}\right]\right. \\
& \left.-2 \cos (q e) \sin c\left[(\Delta k-q) \frac{e}{2}\right] \sin c\left[(\Delta k+q) \frac{e}{2}\right]\right\},
\end{aligned}
$$

where $\Delta k$ is the exchanged photon wavevector. The dependence on the Ge layer thickness $e$ results from the photoelastic profile $p(z)$, the cardinal sinus $\operatorname{sinc}(Q)=\sin (Q) / Q$ being related to the Fourier transform of the rectangular-like photoelastic profile $p(z){ }^{7}$ The Stokes RB spectrum is dominated by the first term in Eq. (1) (the one related to the cardinal sinus being centered at $q=\Delta k$ ). It yields rapidly decaying peaks, with a $2 \pi / e$ wavevector pseudo-periodicity. ${ }^{7}$ Eq. (1)

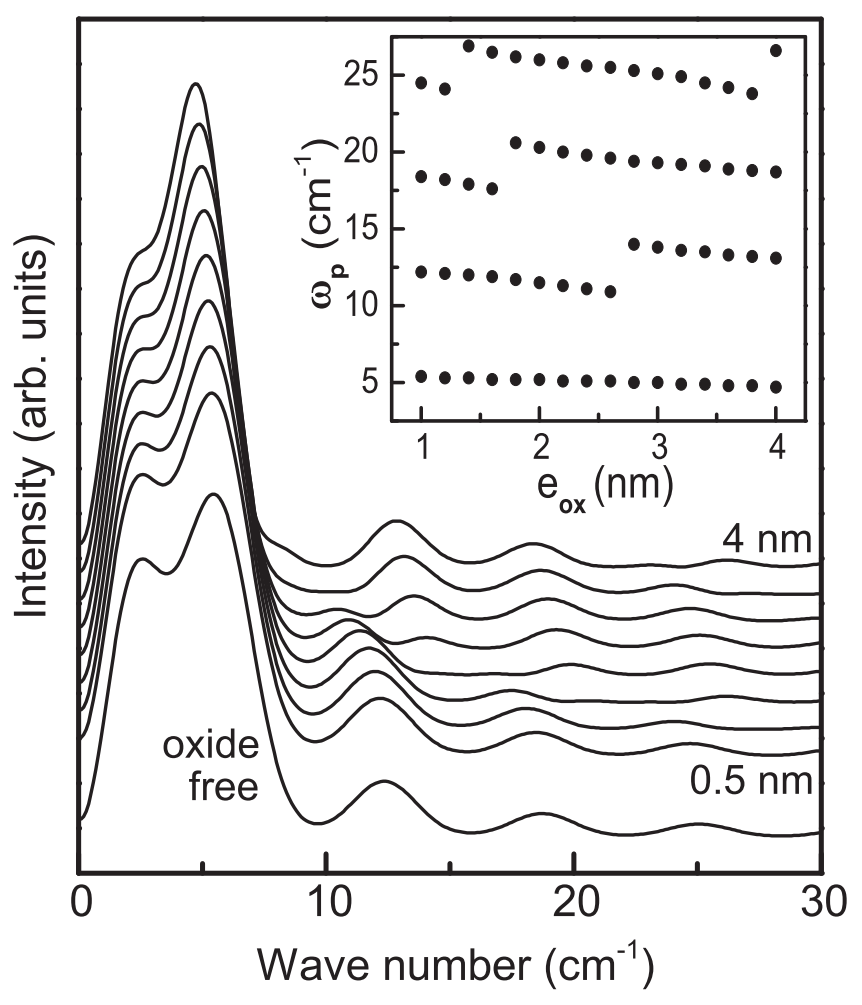

FIG. 2. Spectra calculated for a $25.5 \mathrm{~nm}$ Ge layer: Oxide free case and a series with $e_{o x}$ ranging from $0.5 \mathrm{~nm}$ to $4 \mathrm{~nm}$ with $0.5 \mathrm{~nm}$ steps. Inset: Peak positions $\omega_{p}$ versus $e_{o x}$. 
and the full calculation (bottom spectrum in Fig. 2) do yield the same peak positions. The RB spectral response is related to the photoelastic profile.

From now on, we consider the Ge layer to behave as a RB scattering source, enabling us to investigate the characteristics of an oxide layer being located on top of it and not having its own RB signal. As cavities play roles in this new configuration, the general formulation of the photoelastic model $^{7}$ is required. Fig. 2 shows a series of spectra calculated with the $\mathrm{GeO}_{2}$ oxide thickness $e_{o x}$ ranging from $0.5 \mathrm{~nm}$ to $4 \mathrm{~nm}$, with $0.5 \mathrm{~nm}$ steps. Adding oxide on top of the Ge layer modifies significantly the RB spectra. When increasing progressively $e_{O x}$, some peaks fade out whereas others appear. The inset in Fig. 2 shows that increasing $e_{o x}$ lowers the peak positions. Fig. 2 evidences that RB scattering has a true sensitivity to the oxide presence and thickness.

The dependence on $e_{O x}$ is mainly due to acoustic and photoelastic effects. Regarding the boundary conditions for acoustic displacement fields, the free surface now corresponds to the upper oxide surface. Consequently, with respect to the Ge layer, and thus the photoelastic profile $p(z)$, the acoustic displacement fields are progressively dephased when increasing $e_{O x}$. Conditions yielding maximums in the $\mathrm{RB}$ response therefore change: Peaks are observed at different wavenumbers. Similar capping layer effects on the RB scattering were pointed out earlier for ultrathin superlattices and quantum dot layers. ${ }^{12,13}$

The experimental spectrum (circles) is reported in Fig. 3. Within the $10-30 \mathrm{~cm}^{-1}$ range, it displays three well defined peaks. The calculated spectrum displayed with a

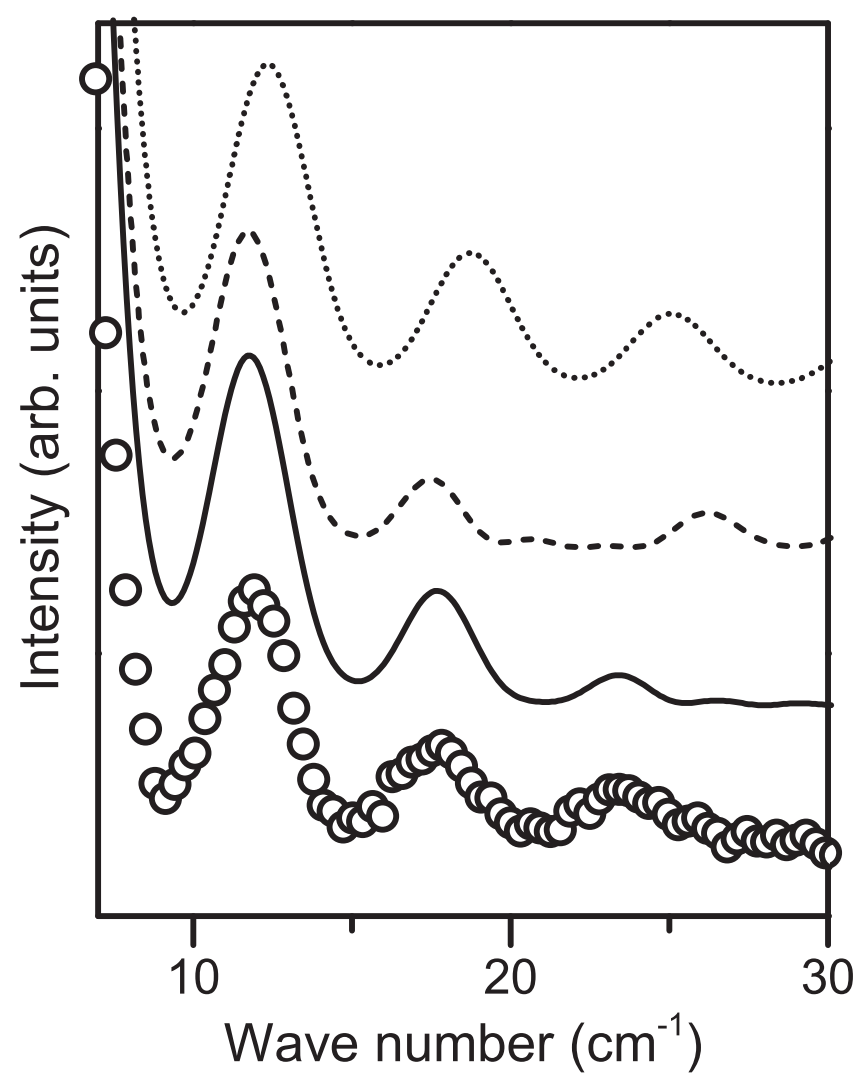

FIG. 3. Experimental spectrum (circles) and calculated ones for a $25.5 \mathrm{~nm}$ Ge layer: Oxide free (dotted line), $1.5 \mathrm{~nm}$ oxide (dashed line), and $0.5 \mathrm{~nm} \mathrm{IL}$ plus $1 \mathrm{~nm} \mathrm{GeO}_{2}$ (solid line). dotted line corresponds to a $25.5 \mathrm{~nm}$ Ge layer and oxide free surface. Three peaks are observed, appearing however at frequencies higher than the experimental ones. According to Fig. 2, adding a thin surface oxide modifies significantly the spectral response. Exploring the $e_{o x}$ thickness range, the best agreement with experiment was found for $e_{o x}=1.5 \mathrm{~nm}$ (dashed line in Fig. 3). Indeed, the first two peaks are well accounted for. Above $20 \mathrm{~cm}^{-1}$, the calculation is however not satisfactory. At the position of the third experimental peak, the calculation is almost flat. Acoustic waves with short wavelengths $\lambda_{a c}$ are very sensitive to changes at small length scales. At $23 \mathrm{~cm}^{-1}$, i.e., at the third peak position in experiment, the acoustic phonon has a $\lambda_{a c}=7 \mathrm{~nm}$ acoustic wavelength in Ge. As $\lambda_{a c}$ corresponds to a $2 \pi$ phase change, a $0.5 \mathrm{~nm}$ change in thickness is equivalent to a significant phase change of $0.44 \mathrm{rad}$. This suggests that such high frequency acoustic waves are able to detect changes on $\mathrm{nm}$ and even sub-nm scales. This motivated us to explore further details, going beyond the structural analysis provided by HRTEM. We found that good agreement between simulation and experiment can be achieved by including an ultrathin Interfacial Layer (IL), in between the $\mathrm{GeO}_{2}$ oxide and $\mathrm{Ge}$ layers. The spectrum displayed with a solid line in Fig. 3 was calculated considering a $0.5 \mathrm{~nm}$ IL. For the sake of simplicity, we interpolated its properties between those of $\mathrm{GeO}_{2}$ and $\mathrm{Ge}$. On top of the IL, we considered a $1 \mathrm{~nm} \mathrm{GeO}$ layer. When the IL is included, whereas the positions of the two first peaks do almost not change, significant changes occur at higher frequencies. In particular, the third peak is now located at $23 \mathrm{~cm}^{-1}$. This simulation accounts thus well for the experimental spectrum. According to the RB simulations, the experimental spectrum is consistent with the following structure: On top of the InGaAs substrate, one has a $25.5 \mathrm{~nm}$ Ge layer, a $0.5 \mathrm{~nm} \mathrm{IL}$, and $1 \mathrm{~nm} \mathrm{GeO}{ }_{2}$ layer. The sum of the thicknesses of the IL and oxide equals $1.5 \mathrm{~nm}$. This is consistent with the amorphous layer shown in Fig. 1. It is interesting to point out that $\mathrm{Ge} / \mathrm{GeO}_{2}$ band offsets were calculated using hybrid density functionals by addressing the interface through a $0.6 \mathrm{~nm}$ suboxide transition region. ${ }^{4}$ The thickness of this transition region is consistent with the one of the IL we evidenced here experimentally.

In conclusion, the RB scattering generated in a thin $\mathrm{Ge}$ layer was investigated to probe the $\mathrm{Ge}$ oxide and $\mathrm{Ge} / \mathrm{GeO}_{2}$ interface. As the acoustic phonons involved in the scattering are sensitive to the Ge layer environment, we were able to determine not only the thickness of the native oxide layer on top of it but also its internal structure. Indeed, the presence of an interfacial layer between the Ge layer and $\mathrm{GeO}_{2}$ oxide is revealed. This is of particular relevance to characterize the $\mathrm{GeO}_{2}$ oxide and $\mathrm{Ge} / \mathrm{GeO}_{2}$ interface and complementary to information on the local structure as derived from XPS. Sensitivity in the sub-nm range is demonstrated. High frequency acoustic phonons are especially sensitive to the actual nature of interfaces. Regarding acoustics, the way the acoustic impedance changes across the interface finally determines the acoustic wave reflections. This study demonstrates that materials not having a significant RB signal can be investigated nevertheless, provided that a layer with a thickness in the nm range is used as an internal probe to generate the RB scattering. 
${ }^{1}$ M. Houssa, A. Satta, E. Simoen, B. D. Jaeger, M. Meuris, M. Caymax, and M. Heyns, in Germanium-Based Technologies (Elsevier, Oxford, 2007).

${ }^{2}$ K. Park, Y. Lee, J. Lee, and S. Lim, Appl. Surf. Sci. 254, 4828 (2008).

${ }^{3}$ K. Prabhakaran and T. Ogino, Surf. Sci. 325, 263 (1995).

${ }^{4}$ P. Broqvist, J. F. Binder, and A. Pasquarello, Appl. Phys. Lett. 94, 141911 (2009); 98, 129901 (2011).

${ }^{5}$ J. F. Binder, P. Broqvist, H. P. Komsa, and A. Pasquarello, Phys. Rev. B 85, 245305 (2012).

${ }^{6}$ A. Mlayah and J. Groenen, in Light Scattering in Solids IX, edited by M. Cardona and R. Merlin (Springer-Verlag, Berlin, Heidelberg, 2007).

${ }^{7}$ J. Groenen, F. Poinsotte, A. Zwick, C. M. Sotomayor-Torres, M. Prunnila, and J. Ahopelto, Phys. Rev. B 77, 045420 (2008).
${ }^{8}$ N. Lou, J. Groenen, G. Benassayag, and A. Zwick, Appl. Phys. Lett. 97, 141908 (2010).

${ }^{9}$ Yu. B. Bolkhovityanov, A. P. Vasilenko, A. K. Gutakovskii, A. S. Deryabin, M. A. Putyato, and L. V. Sokolov, Phys. Solid State 53, 2005 (2011).

${ }^{10}$ M. Levinshtein, S. Rumyantsev, and M. Shur, Handbook Series on Semiconductor Parameters (World Scientific, 1996), Vol. 1.

${ }^{11}$ A. Antoniou and J. A. Morisson, J. Appl. Phys. 36, 1873 (1965).

${ }^{12}$ D. J. Lockwood, M. W. C. Dharma-wardana, G. C. Aers, and J.-M. Baribeau, Appl. Phys. Lett. 52, 2040 (1988).

${ }^{13}$ M. Cazayous, J. Groenen, J. R. Huntzinger, G. Bachelier, A. Zwick, A. Mlayah, E. Bedel-Pereira, F. Negri, H. Carrere, N. Bertru, C. Paranthoen, and O. Dehaese, Phys. Rev. B 69, 125323 (2004). 